\title{
Sepsis Guidelines: Suggestions to Improve Adherence
}

\author{
Niranjan Kissoon, MBBS, FRCP(C), FAAP, FCCM, FACPE \\ Vice President, Medical Affairs \\ BC Children's Hospital and Sunny Hill Health Centre for Children \\ UBC \& BCCH Professor - Global Child Health \\ Disclosures: none
}

Corresponding Author:

Niranjan Kissoon, MBBS, FRCP(C), FAAP, FCCM, FACPE

UBC \& BC Children's

Hospital Professor in Critical Care - Global Child Health

Department of Pediatrics and Emergency Medicine, UBC

604.875.2507

nkissoon@cw.bc.ca

Vancouver, Canada 


\begin{abstract}
The context in which a sepsis guideline is to be used is important and to a large extent determines whether it will be implemented successfully. Factors such as lack of time and resources, lack of reimbursement and organizational constraints may also preclude adoption of guidelines. Thus, sepsis guidelines have been adapted to suit the resources in both resource rich and poor regions of the world. However, even when resources are present, physicians' may not follow guidelines due a myriad of reasons including a lack of agreement with the sepsis guideline or with guidelines in general, as well as lack of motivation and expectancy of the desired outcomes. A holistic approach is necessary to address all issues that may be impediments to guideline adoption and adherence. This approach would include a rigorous transparent method to craft the guideline which includes both clinicians and policy makers and addresses cultural and resource issues.
\end{abstract}

\title{
Running Title
}

Sepsis Guidelines

\section{Key words}

Sepsis, guidelines, outcomes, quality improvement 


\section{Introduction}

Many guidelines have been formulated for the early recognition and treatment of sepsis in children and adults (1-5). However, the context in which a guideline is to be used is important and to a large extent determines whether it will be implemented successfully $(1,2,5-7)$. Thus, sepsis guidelines have been crafted taking into account the resources in both resource rich and poor regions of the world (1-3). For example, sepsis guidelines for children have been adapted to accommodate both resource and skill sets for countries with varying under five mortality rates (Figure 1) and to accommodate resources for monitoring and treatment from district clinics to tertiary care facilities (1) (Figure 2). Such guidelines for adults and children in resource poor areas have also been proposed by the Global Intensive Care Working Group of the European Society of Intensive Care (2). While these efforts are laudable, adherence to these guidelines has met with suboptimal results (adherence rates of $0-30 \%$ ) in both resource poor and rich regions. Therefore, while resources to implement guidelines are important, other factors beyond resources also limit successful adoption.

This manuscript will outline possible reasons for poor compliance and offer some possible solutions to improve compliance with and sustainability of guideline adherence.

\section{Obstacles Resulting in Poor Adherence}

Major contributors to physicians' poor adherence to guidelines include failure to recognize sepsis as a disease, lack of familiarity or lack of awareness of their institution's sepsis guideline, lack of agreement with the sepsis guideline or with guidelines in general, as well as lack of motivation and expectancy of the desired outcomes $(8,9)$. In addition there are many external 
barriers to guideline implementation. For instance, the characteristics of the guidelines may render it impractical to implement - in some cases they are too detailed, while in others they may suggest the use of resources such as laboratory tests, methods of monitoring and treatment options that are not available locally $(2,5,6)$. Local factors such as lack of time, lack of resources, lack of reimbursement and organizational constraints may also preclude adoption of guidelines. For instance, in areas where there are critical staff shortages, it is unreasonable to place increased burdens such as frequent monitoring and documentation which may be the standard of care in areas with substantially more resources. In addition, in many areas of the world, laboratory capabilities for white blood counts to determine SIRS criteria, blood cultures for identification of pathogens and pulse oxymetry to document hypoxia or supplemental oxygen for treatment are not readily available $(5,6)$. Thus guidelines suggesting the use of these resources for case finding and management of sepsis, where they are not available, will not be used.

Poor adoption may also be because incentives may not be aligned to the intended behavior. For instance, using the guideline may identify potential cases of sepsis which necessitates extra time and attention, and the guideline is less likely to be used if productivity and reimbursement are based on throughput only. There are also concerns which lead to skepticism that guidelines may be subject to biases and used as a financial and marketing tool.

A major reason for poor adherence can be due to inherent flaws is the process used in preparing the guidelines. If the process in producing the guideline is viewed as biased or flawed, the best strategies used in implementation is likely to be derailed and lead to low compliance. 
Doubts about the evidence on which a guideline is based may stem from skepticism of the composition of the panel of experts creating the guidelines. While guideline users could sometimes adjust for these biases, in some cases, the values and goals and conflicts are not explicit enough to allow for any adjustments.

Another major concern especially in the United States is the fact that these guidelines may be turned into performance measures to critique the quality of physician care and even dictate hospital accreditation. Moreover, if quality control indicators for evaluation and monitoring are not appropriate and agreed to, monitoring will be inadequate to provide meaningful information. This poses a problem in that if outcome measures are not meaningful, it is very difficult to determine the effectiveness of the guideline and to revise the guideline or address deficiencies in guideline implementation. Another area which has hindered adoption and sustainability is a failure to share the results widely and regularly with team members.

In our local experience, clinicians were skeptical when a sepsis guideline was initially introduced because they felt that screening for sepsis in the emergency department was not necessary because their triage system was robust enough to detect sepsis. Others felt their pediatric early warning systems served the same purpose on the wards while others felt that introduction of the sepsis guideline implied that they were managing sepsis incorrectly beforehand. These reasons for skepticism are not unique to any single institution or any particular guideline and imply that crafting a resource appropriate guideline is an important process however, attention to cultural and legacy issues during implementation are equally important. (Figure 3), (10). 


\section{Solutions to Improve Adherence}

A holistic approach is necessary to address all issues that may be impediments to guideline adoption and adherence (Fig 4). Failure to address all aspects is likely to lead to failure. A robust framework for crafting the guideline and an engagement strategy is an excellent starting point.

\section{Use a Framework for Crafting a Sepsis Guideline}

In order to circumvent some of the barriers outlined above, as a first step, the guideline formulating process should be rigorous and transparent. It is important that the relevant clinicians and policy makers be involved early in the discussion even if an institution decides to craft a guideline denovo or modify or adopt an existing guideline such as the World Federation of Pediatric Intensive and Critical Care Societies or Surviving Sepsis Campaign guidelines $(1,3)$. Failure to invite appropriate and broad representation to the table will likely lead to frustration, suspicion and ultimately failure of implementation. For instance, in our institution an oversight on our part was failure to involve pharmacy at the start of the process even though they are involved in stocking unit doses of antibiotics in the emergency and intensive care units.

The AGREE tool is an example of a tool that provides a roadmap to either create or evaluate a guideline ( http://www.agreetrust.org). Strict adherence to its elements will enable involvement of the appropriate team members, and will ensure transparency in all phases including literature review. It provides the framework for evaluation of resources needed and outcome measures as well as opportunities for revising the guideline. It consists of 6 domains 
(Table 1) which addresses all aspects of formulation and implementation. Each of these domains relates to a series of items (total 23) which guides every step of guideline development and addresses factors that are necessary for success. AGREE is not the only tool that serves to assist in guideline development but it is validated, easy to use, widely accepted and comes with an easily accessible training manual.

Once in use feedback loops using rapid PDSA (Plan, Do, Study, Act) cycles are important to continuously improve the guideline itself as well as address cultural, resource and care process issues that are perceived as barriers. In addition, with the advent of new knowledge and technology, the framework used should incorporate processes to include changes to improve the care processes and outcomes (Figure 3).

\section{Ensure Resources are Available}

In many parts of the world poor guideline adherence is due to a lack of resources. The obstacles in sepsis guideline implementation are unique to the local environment and hence an environmental scan is important to highlight the deficiencies that need to be addressed (6). In some areas these are obvious and mostly relate to a lack of personnel and supplies. For instance, in many areas of the world, human resources and equipment and supplies such as antimicrobials, fluids and oxygen are lacking or sporadically available. Deficiencies in essential staff, equipment and supplies therefore should be brought to the attention of clinicians in positions of authority and policymakers and needs to be addressed for successful implementation $(11,12)$. However, there are limits to resources which can be invested and 
hence this also highlights the importance of crafting guidelines that are realistic to the local context. For instance, expectations regarding laboratory monitoring for diagnosis and response to therapies are context dependent; in many areas of the world, blood counts are rarely available and monitoring may be done using vital signs and pulse oxymetry only.

Overcoming some of the major challenges also requires creativity especially when resources are limited. For example, lack of time and staff is a major barrier which can be somewhat circumvented but creating standard operating procedures $(4,8,9-12,13)$. For instance, sepsis screening should be incorporated into the triage process in emergency departments rather than be viewed as an additional task. In as much as possible, sepsis screening and treatment must be standard work and hence should also be incorporated as an essential screen when deterioration of patients is detected using early warning scores and systems (14). In addition, creating sepsis carts and standard flow sheets would also assist in standardizing and avoiding duplication of work. Prepacked kits consisting of intravenous cannulae and fluid administration set as well as readily accessible essential drugs and fluids may also encourage greater compliance. Specialized equipment is also an issue but in resource limited environments less invasive monitoring and treatment such as use of peripheral inotropes may be needed.

\section{Address Limitations in Skills and Education}

Lack of education including recognition of signs and symptoms of sepsis is an issue that may lead to poor compliance and needs to be addressed. With little training even patients and families, village health workers and non-physician clinicians such as anesthetic assistants and nurses can be taught to recognize and treat sepsis as reported from Malawi $(11,12)$. Familiarity 
with the guideline should be ensured as well as ensuring that several versions are not in circulation. Courses that are sanctioned by the World Health Organization such as the Emergency Triage Assessment and Treatment and the Integrated Management of Adolescent and Adult Illness are useful in resource poor areas and addresses critical illness as well as sepsis $(5,15)$. In some areas procedural skills such as obtaining vascular access and methods to deliver supplemental oxygen are lacking and need to be taught. Large visual displays of the guideline as a constant reminder with easily accessible checklists in the patient care areas are also useful to improve adherence.

\section{Understand and Address Cultural Barriers}

The major hurdles to guideline adherence are attitudes and cultural aversion to guideline adoption and adherence (Figure 4). These barriers can be minimized if meticulous attention is paid to the issues during the early stages when the guidelines are being formulated. Getting the right team at the outset, identifying champions and addressing resource limitations goes a long way in changing negative cultural perceptions and attitudes to guidelines. In addition, to overcome this barrier, advocacy, leadership and support is necessary from clinicians and policy makers alike to address all facets of the process of formulating and introducing the guideline. We have found that involvement of an anthropologist to assist in identifying causes of aversion and to facilitate change can enable dramatic positive gains. Attempts at building a community of practice emphasizing shared values and goals and shared learning experiences can also be useful robust enabler. A community of practice may generate innovative ideas to circumvent resource limitations, ensure staff engagement and accelerate educational efforts $(16,17)$. For 
instance, a community of practice can foster collaboration among medical disciplines such as the emergency and intensive care personnel whereby major obstacles in seamless care can be circumvented through standard operating practices. Indeed, a team model is associated with an increase in compliance with guidelines of $80 \%$ versus $40 \%$ in the non-team model (7). The community can also foster early referral to the intensive care unit and rapid response team's involvement in the care of patients with sepsis.

\section{Ensure Sustainability of Gains}

A stewardship program can enable robust quality assurance and the designation of an ambassador and use of feedback systems may facilitate sustainability $(16,18)$. Ultimately, ensuring guideline adherence requires a gargantuan effort from those in leadership such that the culture of the organization embraces guidelines as part of standard work. Moreover, commitment has to be ongoing because a decline in vigilance is likely to result in loss of previous gains (4). Thus, sharing of data and outcomes regularly with all team members and facilitating processes to improve is required. The rewards for sustained dogged effort can be improved guideline adherence both in resource-rich and resource- poor environments.

\section{Conclusion}

Guidelines are useful in improving the quality of care and outcomes, reducing inappropriate variation in practice and promoting efficient use of resources. However, the benefits are minimized by poor adoption in both resource rich and resource poor environments. In order to improve adoption a uniform and transparent inclusive process is needed to formulate and introduce the guidelines. An environmental scan to determine to identify and address the 
barriers to implementation is also important. The most common barriers which hinder adoption are cultural and lack of resources. These barriers should be addressed early in the implementation stage for guideline adoption to be successful. Ensuring that a guideline is successfully adopted requires a tremendous investment of resources and effort. However, favorable outcomes associated with guidelines far outweigh the effort that is needed for successful implementation. 


\section{References}

1. Kissoon N, Carcillo JA, Espinosa V et al. (2011) World Federation of Pediatric and Intensive Care Societies: Global Sepsis Initiative. Pediatr Crit Care Med. 12(5):494-503.

2. Dunser MW, Festic E, Dondorp A et al. (2012) Recommendations for sepsis management in resource-limited settings. Intensive Care Med. 38(4):557-74.

3. Dellinger RP, Levy MM, Rhodes A et al. (2013) Surviving Sepsis Campaign: international guidelines for management of severe sepsis and septic shock 2012. Intensive Care Med. 39(2):165-228.

4. Plambech MZ, Lurie Al, Ipsen HL. (2012) Initial successful implementation of sepsis guidelines in an emergency department. Dan Med J. 59(12):A4545.

5. Jacob ST, Lim M, Banura P et al. (2013) Integrating sepsis management recommendations into clinical care guidelines for district hospitals in resource-limited settings: the necessity to augment new guidelines with future research. BMC Medicine 2013, 11:107.

6. Baelani I, Jochberger S, Laimer T et al. (2012) Identifying resource needs for sepsis care and guideline implementation in the Democratic Republic of the Congo: a cluster survey of 66 hopsitals in four eastern provinces. Middle East J Anesthesiol. 21(4):559-75.

7. Na S, Kuan WS, Mahadevan M et al. (2012) Implementation of early goal-directed therapy and the surviving sepsis campaign resuscitation bundle in Asia. Int J Qual Health Care. 24(5):452-62.

8. Cabana MD, Rand CS, Powe NR et al. (1999) Why don't physicians follow clinical practice guidelines: A framework for improvement. JAMA 282(15):1458-65. 
9. Shaneyfelt TM, Centor RM. (2009) Reassessment of clinical practice guidelines: go gently into that good night. JAMA. 301(8):868-9.

10. Leone M, Ragonnet B, Alonso S et al. (2012) Variable compliance with clinical practice guidelines identified in a 1-day audit at 66 French adult intensive care units. Crit Care Med. 40(12):3189-95.

11. Pollach G, Namboya F. (2013) Preventing intensive care admissions for sepsis in tropical Africa (PICASTA): and extension of the international pediatric global sepsis initiative: and African perspective. Pediatr Crit Care Med. 14(6):561-70.

12. Kissoon N. (2013) Preventing intensive care admissions for sepsis in tropical Africa: PICASTA - food for thought. Pediatr Crit Care Med. 14(6):644-5.

13. Barochia AV, Cui X, Vitberg D et al. (2010) Bundled care for septic shock: an analysis of clinical trials. Crit Care Med. 39(2):668-78.

14. Nguyen HB, Corbett SW, Steele R et al. (2007) Implementation of a bundle of quality indicators for the early management of severe sepsis and septic shock is associated with decreased mortality. Crit Care Med. 35(4):1105-12.

15. Irimu GW, Gathara D, Zurovac D et al. (2012) Performance of health workers in the management of seriously sick children at a Kenyan tertiary hospital: before and after a training intervention. PLoS One. ;7(7):e39964Epub 2012 Jul 31.

16. Schramm GE, Kashyap R, Mullon JJ et al. (2011) Septic shock: a multidisciplinary response team and weekly feedback to clinicians improve the process of care and mortality. Crit Care Med. 39(2):252-8. 
17. Wenger E. (2000) Communities of practice and social learning systems. Organization. $7(2): 225-46$.

18. Shorr AF, Micek ST, Jackson WL et al. (2007) Economic implications of an evidencebased sepsis protocol: can we improve outcomes and lower costs? Crit Care Med. 35(5):1257-62. 


\section{Figure Legend}

Figure 1

Global Newborn and Child Sepsis Guidelines: Proposed Bundles A-D align with local resources

Figure 2

Resources and interventions expected to be made available at different levels of health care facilities in resource-poor economies

Figure 3

Clinical Practice Guidelines: Preparation, Implementation, Evaluation and Revision are all important for successful adoption

Figure 4

Improving Performance in Sepsis Management requires attention to both process and structure. Individuals, the environment and infrastructure must be conducive to successful guideline adoption. 


\section{Table 1}

The Appraisal of Guidelines for Research and Evaluation (AGREE)

- Domain 1. Scope and Purpose: the overall aim of the guideline, the specific health questions, and the target population

- Domain 2. Stakeholder Involvement: the extent to which the guideline was developed by the appropriate stakeholders and represents the views of its intended users

- Domain 3. Rigor of Development: the process used to gather and synthesize the evidence, the methods to formulate the recommendations, and to update them

- Domain 4. Clarity of Presentation: the language, structure, and format of the guideline

- Domain 5. Applicability: the likely barriers and facilitators to implementation, strategies to improve uptake, and resource implications of applying the guideline

- Domain 6. Editorial Independence: the formulation of recommendations not being unduly biased with competing interests

- Overall assessment: Includes the rating of the overall quality of the guideline and whether the guideline would be recommended for use in practice. 